TecnoHumanismo. Revista Científica

https://doi.org/10.53673/th.v1i3.62

Abril 2021

Volumen 1 / No. 03

ISSN: 2710-2394

pp.111-128

https://tecnohumanismo.online

\title{
Impacto de volatilidad del tipo de cambio del dólar en las monedas de países latinoamericanos
}

Impact of the volatility of the dollar exchange rate on the currencies of Latin American countries

Impacto da volatilidade da taxa de câmbio do dólar nas moedas dos países latino-americanos

\section{ARTÍCULO ORIGINAL}

\section{César Loo Gil}

cesarloo@biofab.com.pe

https://orcid.org/0000-0001-8396-5972

Científico Investigador de BioFab Inc.y del Centro de Investigación \& Producción Científica IDEOs

Recibido 28 de Setiembre 2021 | Arbitrado y aceptado 28 de Setiembre 2021 | Publicado en 01 Octubre 2021

\section{RESUMEN}

El objetivo del presente estudio fue determinar el impacto de la volatilidad del tipo de cambio del dólar frente a las monedas de los países latinoamericanos, tomando como casos de estudio las situaciones en Perú, Chile, Colombia y Brasil. El estudio fue de tipo observacional, descriptivo, transversal y no experimental. El periodo el cual se tomó en cuenta principalmente para el presente análisis, fue el primer y segundo trimestre del año 2021, por lo que el estudio realizado se determinó por medio de los datos existentes dentro del plazo mencionado. Se analizaron las razones del por qué el tipo de cambio del dólar impacta en la economía de los países y las situaciones actuales que afectan la volatilidad de la moneda estadounidense fuera de sus fronteras. Los resultados demostraron que el alza del dólar tiene una implicancia en la moneda de los países estudiados, la cual se refleja en un incremento del precio de los productos importados a causa de la devaluación de la moneda local frente al dólar, debido, principalmente, a las incertidumbres políticas y en base a la pandemia Covid-19, que estos países presentan.

Palabras Clave: Dolarización, Latinoamérica, Tipo de cambio

\begin{abstract}
The objective of this study was to determine the impact of the volatility of the dollar exchange rate against the currencies of Latin American countries, taking as case studies the situations in Peru, Chile, Colombia and Brazil. The study was of an observational, descriptive, cross-sectional and non-experimental type. The period which was mainly taken into account for the present analysis, was the first and second quarters of the year 2021, so the study carried out was determined by means of the existing data within the aforementioned period. The reasons why the dollar exchange rate impacts the economy of the countries and the current situations that affect the volatility of the US currency outside their borders were analyzed. The results showed that the rise in the dollar has an implication on the currency of the countries studied, which is reflected in an increase in the price of imported products due to the devaluation of the local currency against the dollar, mainly due to the political uncertainties and based on the Covid19 pandemic, that these countries present.
\end{abstract}

Keywords: Dollarization, Latin America, Exchange rate

\section{RESUMO}

O objetivo deste estudo foi determinar o impacto da volatilidade da taxa de câmbio do dólar em relação às moedas de países latinoamericanos, tomando como estudos de caso as situações do Peru, Chile, Colômbia e Brasil. O estudo foi do tipo observacional, descritivo, transversal e não experimental. O período que foi maioritariamente considerado para a presente análise foi o primeiro e o segundo trimestres do ano de 2021, pelo que o estudo realizado foi determinado com base nos dados existentes no referido período. Foram analisados os motivos pelos quais a cotação do dólar impacta a economia dos países e as situações atuais que afetam a volatilidade da moeda norte-americana fora de suas fronteiras. Os resultados mostraram que a alta do dólar tem implicações na moeda dos países estudados, o que se reflete na alta dos preços dos produtos importados devido à desvalorização da moeda local frente ao dólar, principalmente devido às incertezas políticas e com base na pandemia Covid-19, que esses países apresentam.

Palavras-chave: Dolarização, América Latina, Taxa de câmbio 


\section{INTRODUCCIÓN}

La estabilidad de los precios en los países, son básicamente el objetivo primordial que las autoridades deberían perseguir en el tiempo de sus mandatos. Tener un conocimiento sobre lo que hay detrás de una economía estable y con precios constantes en el tiempo, es vital para plantear estrategias que permitan cumplir tal cuestión. Por ello, a parte de tener en cuenta los factores internos que determinan los niveles de precios dentro de un país, se toma en cuenta también el campo externo, ya que con los años y con el desarrollo de la globalización, la economía de los países se ve afectada también por los sucesos ocurridos en los demás países del mundo.

El tipo de cambio es la forma en que se mide el valor de una moneda con respecto a otra; del dólar de los Estados Unidos de América con, por ejemplo, la moneda sol en Perú, el peso en Colombia, denominado "peso colombiano", o el real en Brasil. El tipo de cambio es reflejado por las variaciones en la oferta y demanda de divisas, a esto se le conoce como un régimen de flotación. Dicha variación está influida, a parte de otros factores, principalmente por la evolución o cambios de los términos de intercambio; es decir, en la relación que hay entre los precios de los productos de exportación y los de precios de los productos de importación; el nivel de comercio exterior, "los flujos de capital y cambios en las decisiones de portafolio de las personas, empresas y bancos.” (Banco Central de Reserva del Perú, 2021)

La medición del poder adquisitivo de una moneda se da en base a la suma de bienes y servicios que puede adquirir esta. Para que se cumpla la PPC, cualquier alteración en el tipo de cambio tiene que ser parecida a la resta entre la inflación del otro país o grupo de países; es decir, la inflación externa, y la inflación interna. El tipo de cambio real es una definición relevante en el campo económico. Esta medida "captura el precio relativo de los bienes y servicios de una economía respecto a otro país o conjunto de países". TCR E*P / P* =, donde: TCR = Tipo de Cambio Real $\mathrm{E}=$ Tipo de Cambio Nominal $\mathrm{P} *=$ Nivel de precios externo $\mathrm{P}=$ Nivel de precios doméstico (Bermúdez, E, 2018).

La inflación puede ser definida como el incremento sostenido y generalizado de los precios de bienes y servicios en un país en un rango de tiempo que es sostenido, (generalmente un año). Cuando los precios a nivel global, suben con cada unidad 
monetaria, se obtiene menor cantidad de bienes y servicios; en otras palabras, la inflación evidencia el descenso del poder adquisitivo monetario: el valor real del medio interno de intercambio y unidad de medición de una economía, significan una pérdida. (Aguilar, J.L., 2018).

La crisis mundial que se vive actualmente a causa de la pandemia por Covid-19 ha generado inestabilidades económicas en todos los países, incluyendo a las grandes potencias como Estados Unidos. El problema se ha reflejado en la volatilidad de la moneda dólar, la cual es la moneda más usada globalmente y la que mayor presencia tiene en la economía de los países.

Así mismo, en los países como Perú, Brasil y Colombia, se han tenido sucesos internos los cuales han tenido impacto directo en su economía. La existencia de manifestaciones por incertidumbre política, el mal manejo por parte de las autoridades frente a la crisis mundial actual, y el panorama nublado que tienen estos países frente a su futuro, ha generado que las inversiones exteriores sean mas rigurosas por el estado de alerta que se encuentran al no encontrar una estabilidad que les permita tener la seguridad de hacer una buena inversión en los países.

Por todo lo antes mencionado, se consideró importante e interesante plantear como objetivo del presente estudio, el determinar el comportamiento del tipo de cambio frente a los países: Perú, Colombia y Brasil, y el impacto en la inflación de estos y en su economía, en el periodo de los dos primeros trimestres del año 2021. 
METODOLOGÍA

El presente estudio es de tipo observacional y descriptivo porque la investigación solo se limita a medir la existencia y distribución de las variables de estudio durante un corte de tiempo. Por otro lado, es de carácter transversal, ya que los datos recolectados y obtenidos fueron observados y descritos, como ya se había mencionado, durante un corte en el tiempo. Además, presenta un diseño no experimental, ya que el investigador no interviene directamente en el objetivo de la investigación (Veiga de Cabo, J., De La Fuente, E. y Zimmermann, M., 2008) y se observan las manifestaciones exactamente "como se dan en su contexto natural" (Bermúdez, E, 2018). Finalmente, se considera de un solo grupo, puesto que no existe un grupo control.

Para llevar a cabo el presente trabajo de investigación, se realizó una búsqueda bibliográfica en diversas fuentes tanto escritas como virtuales tales como: en libros relacionados con las variables de estudio y los conceptos pertinentes que se manejan en el mismo; así como en revistas de especialización de la materia, herramientas virtuales (páginas web, reportes de los diarios electrónicos más representativos de Perú, Chile, Colombia y Brasil, comentarios de expertos en la materia, etc.), artículos científicos, y herramientas informáticas proporcionadas, en el caso de Perú, por el Banco Central de Reserva del Perú (BCRP); con respecto a Chile, por el Banco Central de Chile; en caso de Brasil, por las principales páginas web de economía de su país y finalmente, en lo que respecta a Colombia, por el Banco de la República de Colombia. Asimismo, se extrajeron una serie de datos estadísticos mensuales en las mismas fuentes en base al tipo de cambio en Perú, Chile, Colombia y Brasil. Los criterios que se tomaron en cuenta para el desarrollo del presente estudio se resumieron básicamente en que el período de análisis del tipo de cambio tenía que ser únicamente entre el mes de febrero del año 2021 hasta la actualidad para cada uno de los países estudiados.

Los resultados obtenidos fueron a base de la estadística descriptiva, la que involucra el visualizar, recolectar y describir los datos en base a la problemática del estudio, esto a través de la utilización de gráficos, cuadros y tablas, los cuales fueron obtenidos 
de las fuentes bibliográficas y electrónicas ya antes mencionadas (Bermúdez, E, 2018).

\section{RESULTADOS}

Situación en Perú

Gráfico N¹: Tipo de cambio según pronóstico del I Trimestre (PROYECCIÓN BBVA)

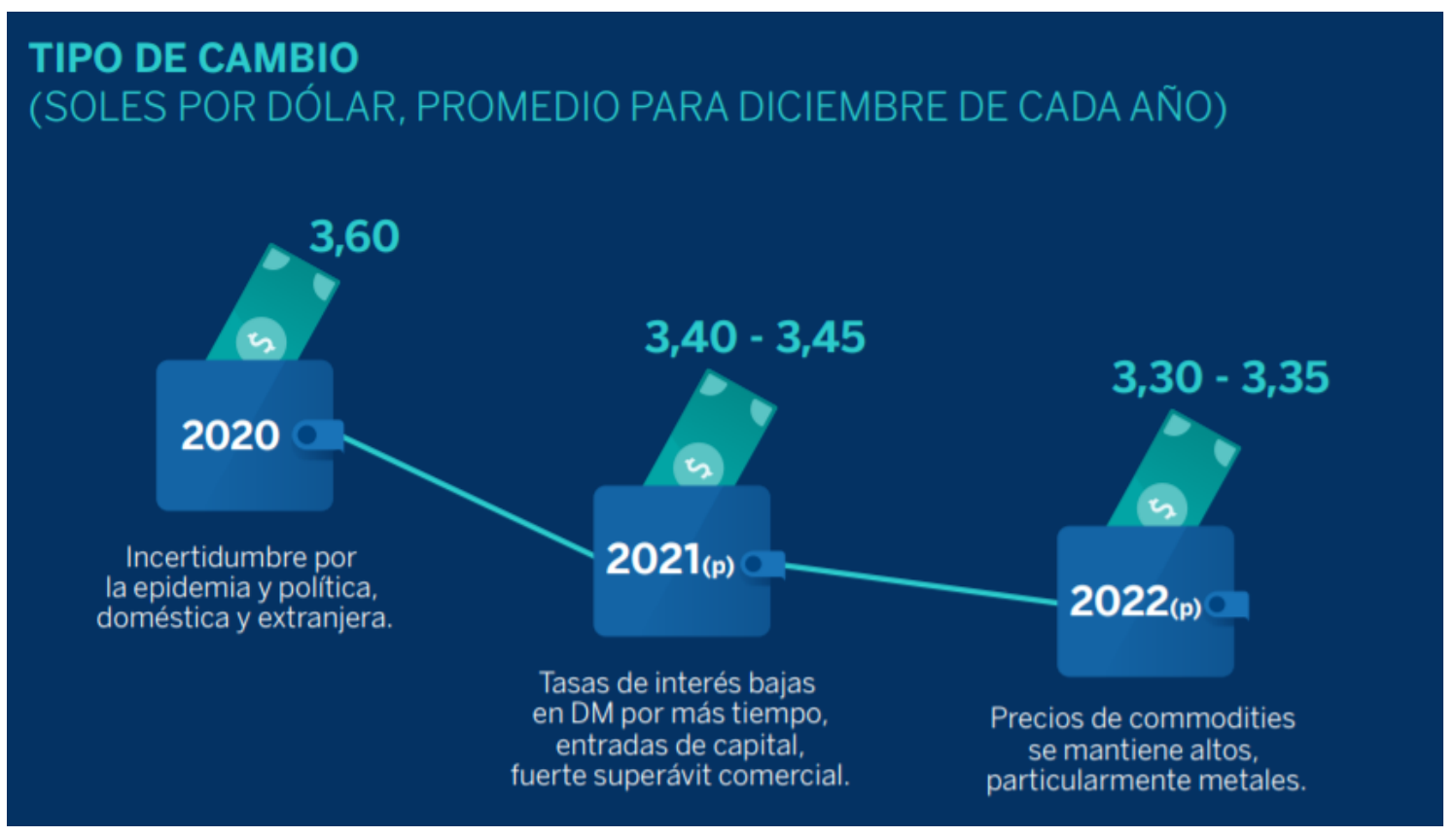

Fuente: Bbva Research (2021)

Grafico N²: Tipo de cambio según pronostico del II Trimestre (PROYECCIÓN BBVA) 


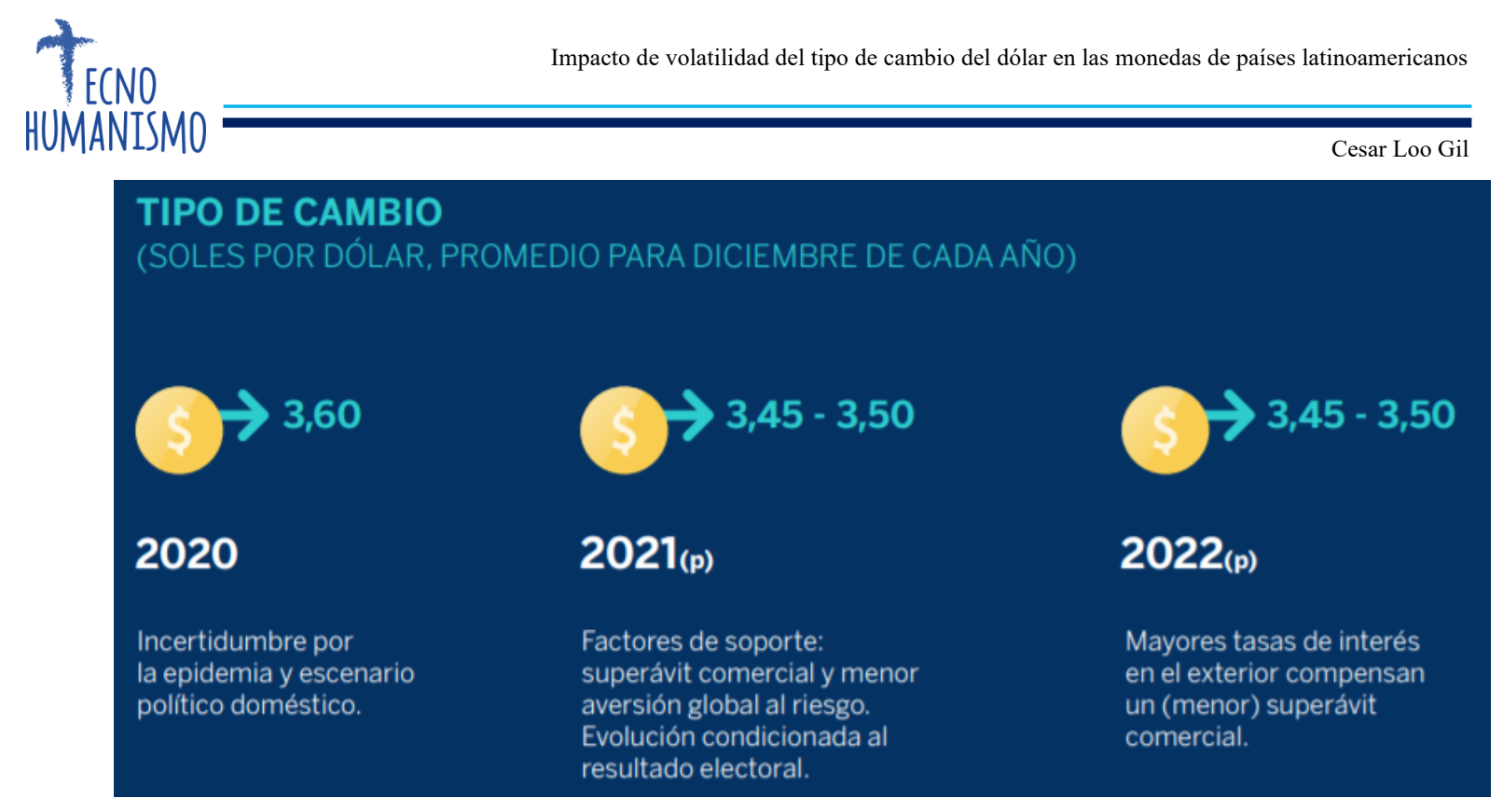

Fuente: Bbva Research (2021)

En los Gráficos $\mathrm{N}^{\circ} 1$ y 2 se puede apreciar la proyección que se tuvo respecto al tipo de cambio en el Perú, se ve que, dentro de los factores considerados para estas proyecciones, se tomaron en cuenta el superávit comercial, la evolución frente a la incertidumbre política debido a los resultados electorales y el nivel de riesgo presente a nivel global. En el primer trimestre se pronosticaba que el dólar estaría entre 3.40 a 3.45 y se veía un panorama en recuperación, además, se plasmaba un fuerte superávit comercial frente a las exportaciones e importaciones, y una entrada de capital como proyección para el siguiente periodo. Sin embargo, en la proyección del segundo trimestre se refleja que el tipo de cambio ha aumentado al rango de 3,45 y 3,55 , y esto es se debe a que en los factores se hace mención a la evolución que pueda tener el tema político en el país, y su implicancia con las medidas que se llegaría a tomar por el gobierno actual.

Grafico N³: Tipo de cambio en Perú en Febrero-Septiembre del 2021 


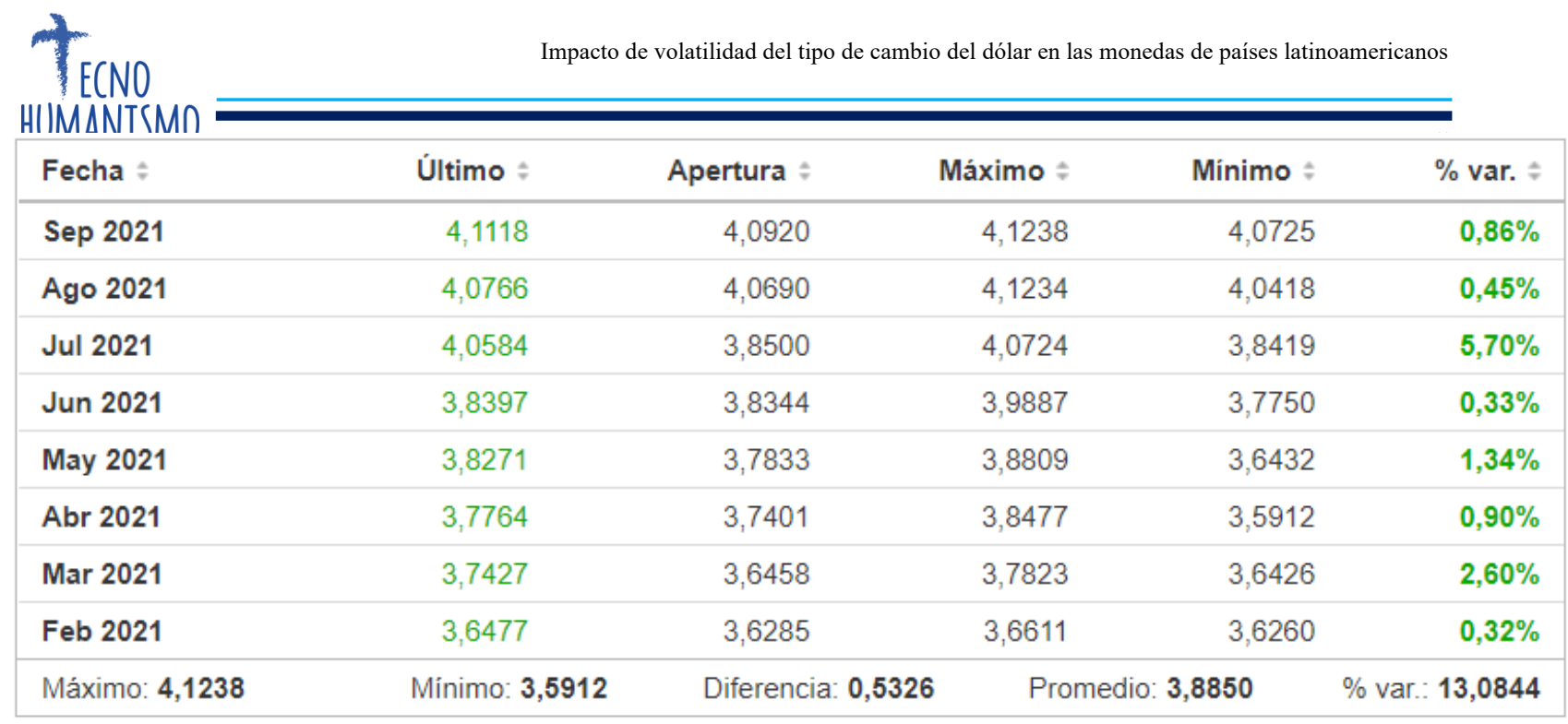

Fuente: Investing (2021)

Se puede observar que se obtuvo una mayor fluctuación al alza, a finales del mes de mayo

y hubo una pequeña baja en el mes de julio; sin embargo, terminó por inclinarse

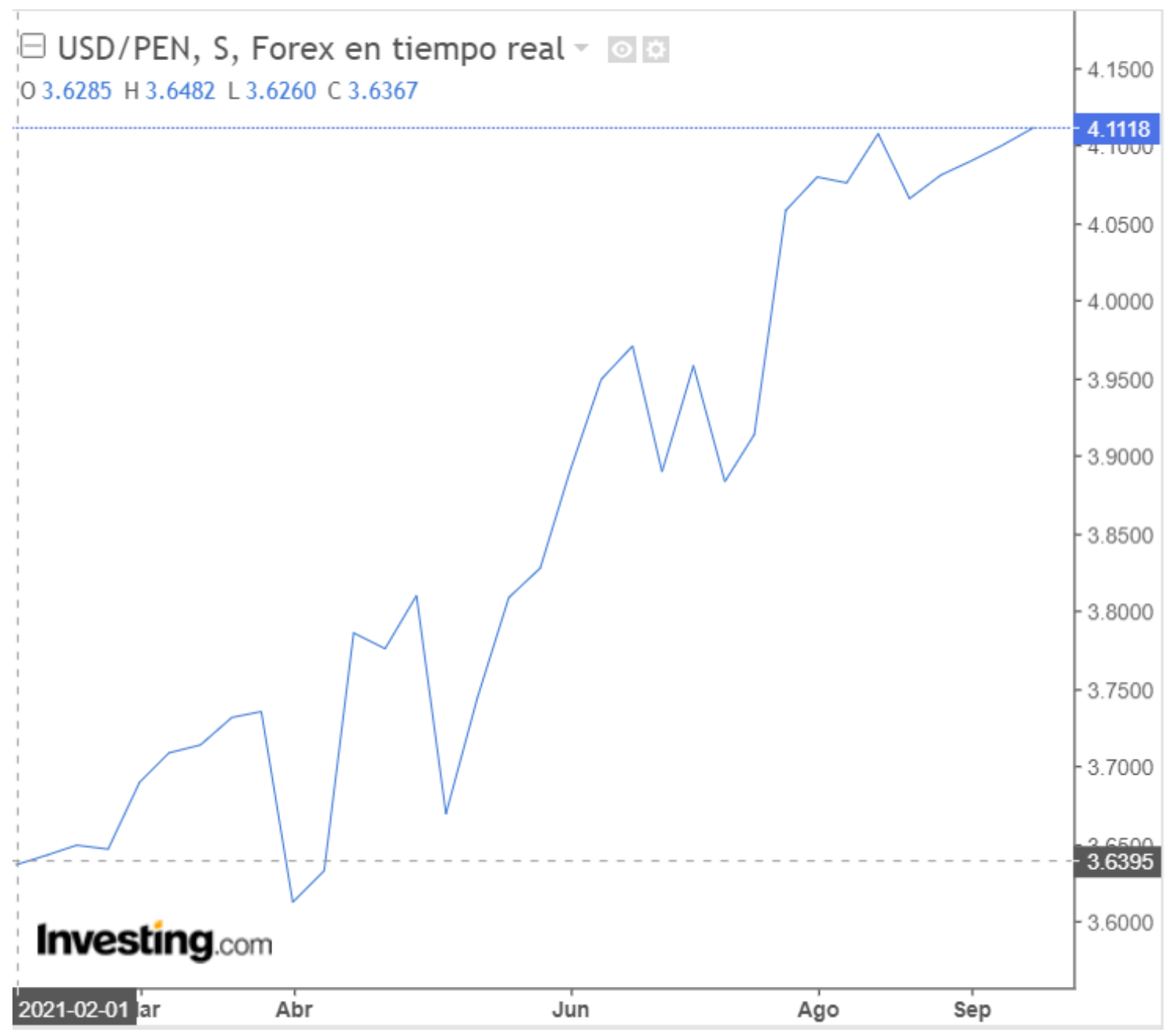

hacia el alza hasta llegar a 4,1118. 
Situación en Colombia

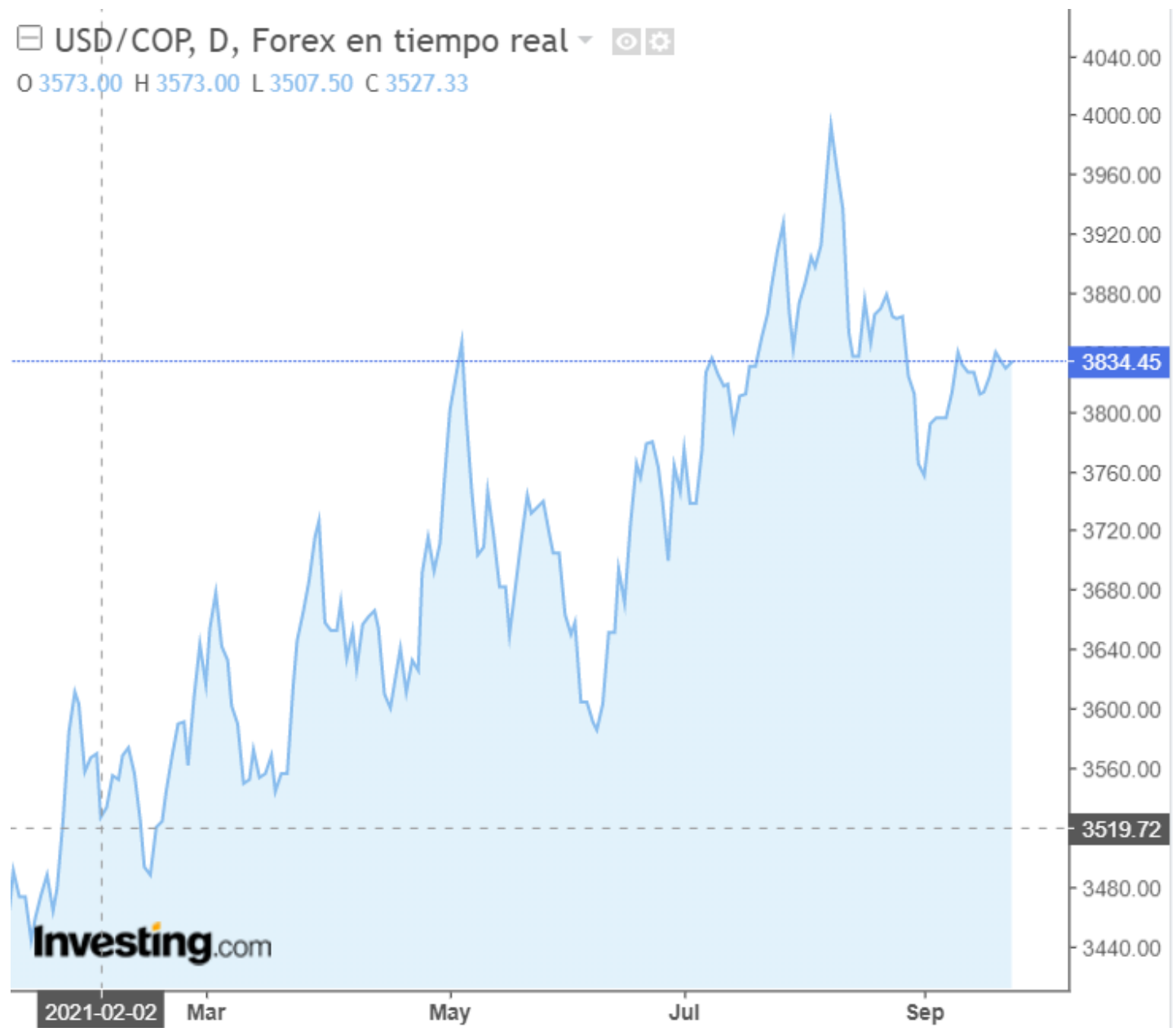

\begin{tabular}{|c|c|c|c|c|c|}
\hline Fecha * & Último * & Apertura $=$ & Máximo $=$ & Mínimo $=$ & $\%$ var. $\neq$ \\
\hline Sep 2021 & $3.835,00$ & $3.769,15$ & $3.859,50$ & $3.739,00$ & $1,81 \%$ \\
\hline Ago 2021 & $3.766,68$ & $3.878,00$ & $4.009,14$ & $3.761,50$ & $-2,77 \%$ \\
\hline Jul 2021 & $3.874,00$ & $3.750,00$ & $3.939,70$ & $3.725,80$ & $3,39 \%$ \\
\hline Jun 2021 & $3.747,00$ & $3.708,00$ & $3.798,00$ & $3.573,00$ & $1,11 \%$ \\
\hline May 2021 & $3.705,75$ & $3.753,30$ & $3.866,75$ & $3.643,35$ & $-1,22 \%$ \\
\hline Abr 2021 & $3.751,43$ & $3.661,70$ & $3.759,05$ & $3.587,55$ & $2,53 \%$ \\
\hline Mar 2021 & $3.658,95$ & $3.647,00$ & $3.755,00$ & $3.515,68$ & $0,40 \%$ \\
\hline Feb 2021 & $3.644,50$ & $3.569,00$ & $3.651,95$ & $3.481,90$ & $2,15 \%$ \\
\hline Máximo: 4.009,14 & Minimo: & Difere & 7,24 & o: $3.747,91$ & var:: 7,49 \\
\hline
\end{tabular}

Gráfico N 4: Tipo de cambio en Colombia en Febrero-Septiembre del 2021

Fuente: Investing (2021) 
Situación en Brasil

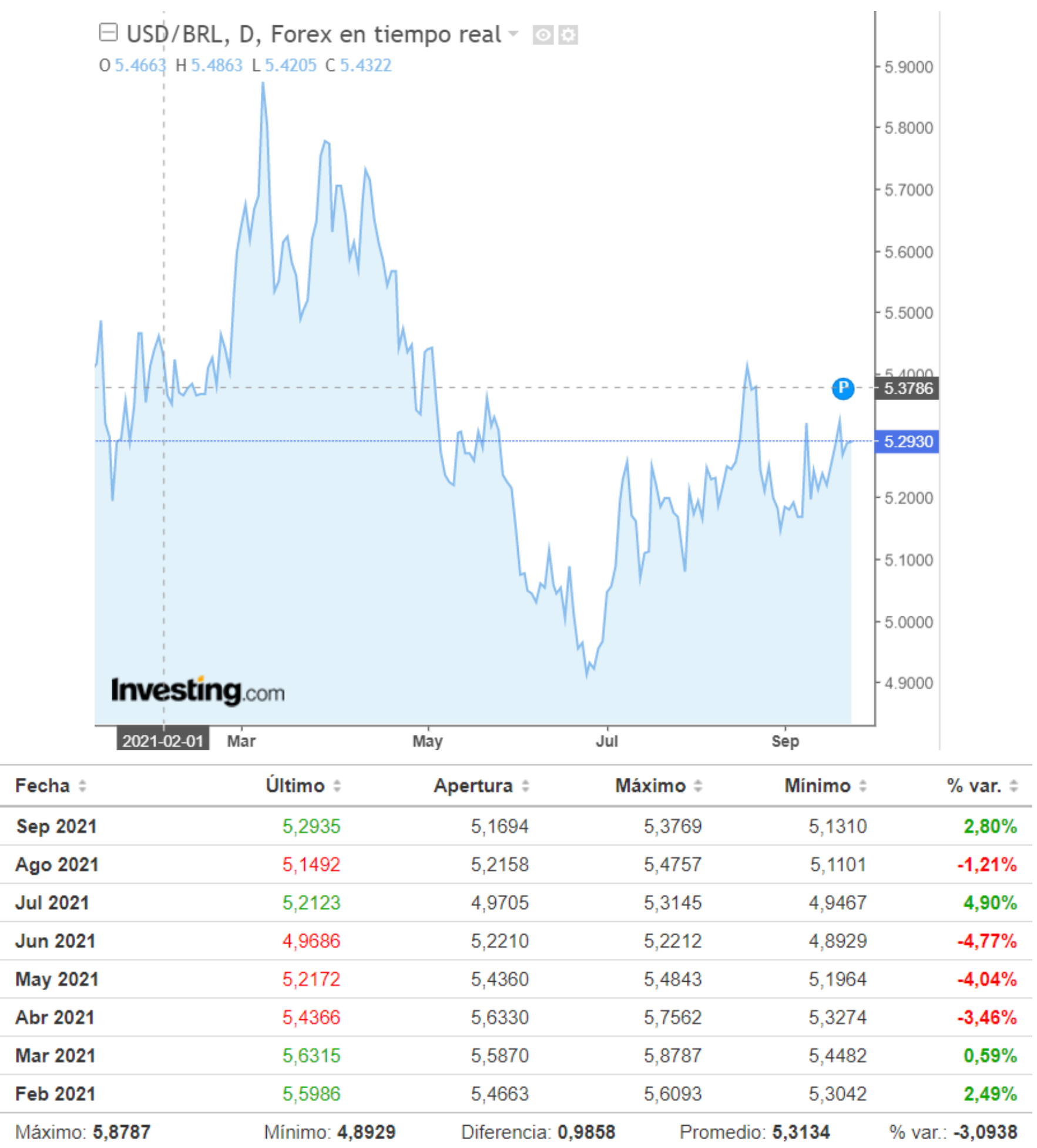

Gráfico $N^{\circ}$ 5: Tipo de cambio en Brasil en Febrero-Septiembre del 2021

Fuente: Investing (2021) 
Situación en Chile

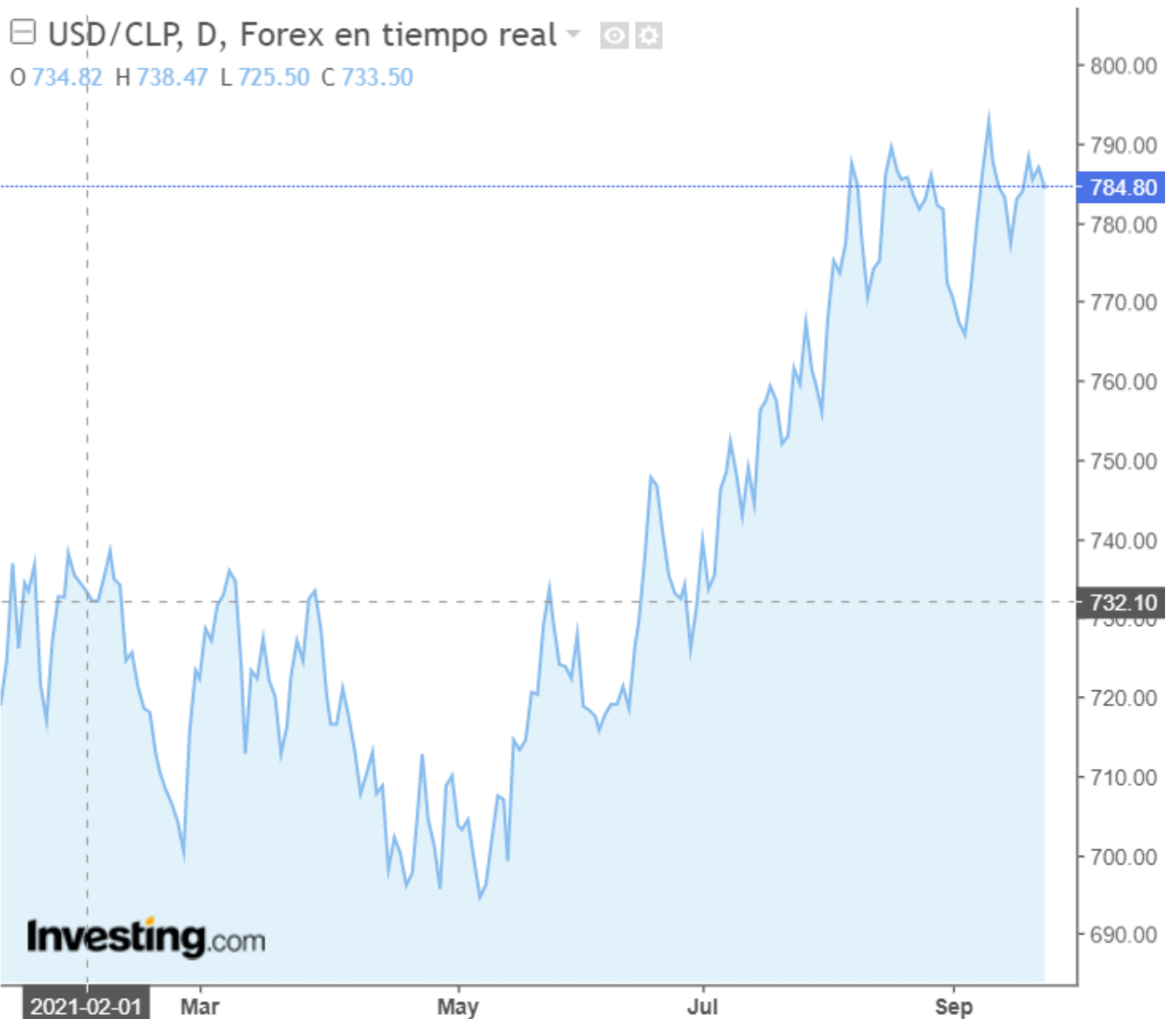

\begin{tabular}{|c|c|c|c|c|c|}
\hline Fecha * & Último * & Apertura $=$ & Máximo $=$ & Mínimo & $\%$ var. $\neq$ \\
\hline Sep 2021 & 784,80 & 773,23 & 794,80 & 762,08 & $1,58 \%$ \\
\hline Ago 2021 & 772,63 & 756,65 & 796,13 & 752,92 & $2,17 \%$ \\
\hline Jul 2021 & 756,20 & 732,30 & 772,91 & 727,42 & $3,35 \%$ \\
\hline Jun 2021 & 731,70 & 722,88 & 757,80 & 713,62 & $1,28 \%$ \\
\hline May 2021 & 722,43 & 710,75 & 738,17 & 690,28 & $1,71 \%$ \\
\hline Abr 2021 & 710,30 & 720,45 & 724,00 & 693,33 & $-1,37 \%$ \\
\hline Mar 2021 & 720,20 & 723,89 & 743,36 & 712,47 & $-0,46 \%$ \\
\hline Feb 2021 & 723,51 & 734,82 & 740,18 & 697,39 & $-1,51 \%$ \\
\hline Máximo: 796,13 & Mínimo: 690,28 & Diferer & 5,85 & dio: 740,22 & var.: 6,84 \\
\hline
\end{tabular}

Gráfico N 6: Tipo de cambio en Chile en Febrero-Septiembre del 2021

Fuente: Investing (2021) 
Gráfico No 7: Cuadro comparativo de la variación porcentual del Tipo de cambio en Perú, Colombia, Brasil y Chile

\section{Variación porcentual}

$20 \%$

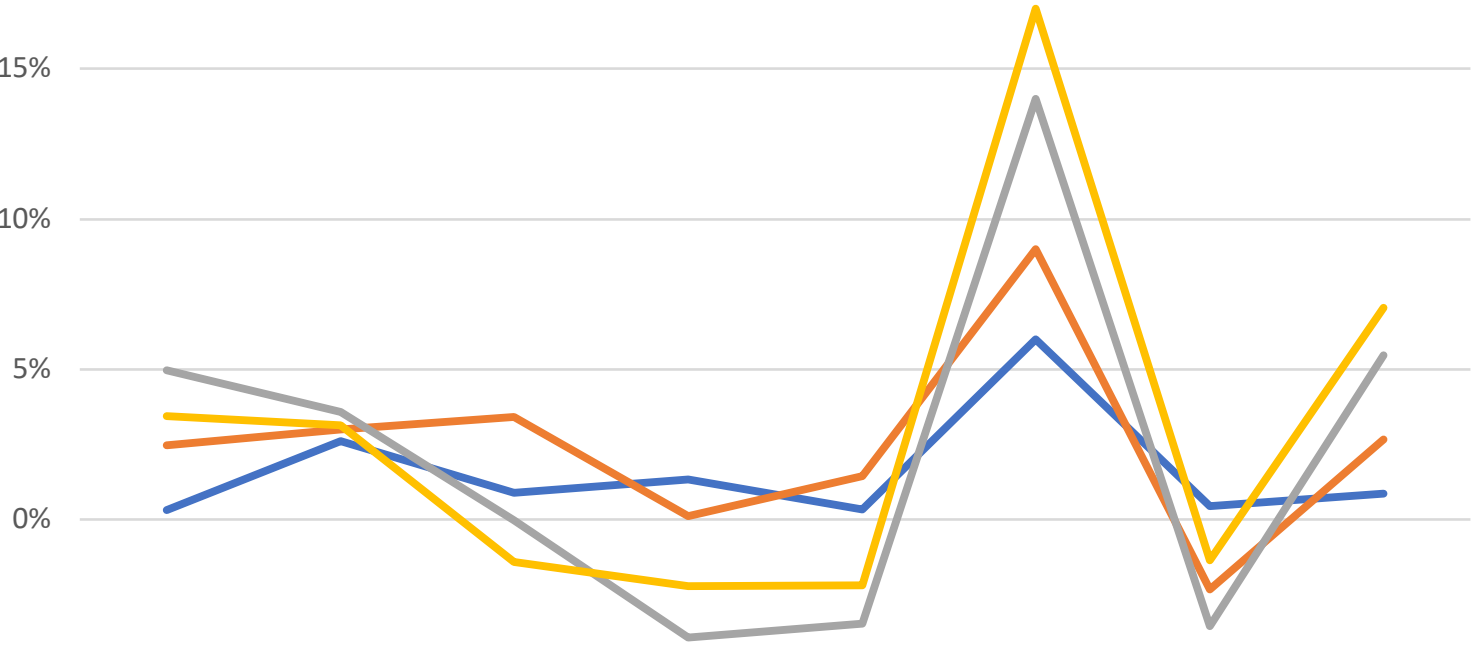

$-5 \%$

Febrero Marzo

Abril

Mayo

Junio

Julio

Agosto

Setiembre

— Perú

Fuente: Elaboración propia 
Gráfico N 8: Cuadro comparativo del Tipo de cambio en Perú, Colombia, Brasil y Chile

\begin{tabular}{|c|c|c|c|c|c|c|c|c|c|c|c|c|}
\hline & Perú & & & Colomb & & & Brasil & & & Chile & & \\
\hline & $\begin{array}{l}\text { apertu } \\
\text { ra }\end{array}$ & ierre & o var & $\begin{array}{l}\text { apertur } \\
\text { a }\end{array}$ & ierre & $\begin{array}{l}\% \\
\text { var }\end{array}$ & $\begin{array}{l}\text { apertur } \\
\mathrm{a}\end{array}$ & cierre & var & $\begin{array}{l}\text { apertur } \\
\text { a }\end{array}$ & cierre & o var \\
\hline $\begin{array}{r}\text { Febrero } \\
2021\end{array}$ & 3,6285 & $\begin{array}{l}3,647 \\
7\end{array}$ & $0,32 \%$ & $\begin{array}{l}3.569,0 \\
0\end{array}$ & $\begin{array}{l}3.644, \\
50\end{array}$ & $\begin{array}{l}2,15 \\
\%\end{array}$ & 5,4663 & 5,5986 & $2,49 \%$ & 734,82 & $\begin{array}{l}723,5 \\
1\end{array}$ & $\begin{array}{l}- \\
1,51 \%\end{array}$ \\
\hline $\begin{array}{r}\text { Marzo } \\
2021\end{array}$ & 3,6458 & $\begin{array}{l}3,742 \\
7\end{array}$ & $2,60 \%$ & $\begin{array}{l}3.647,0 \\
0\end{array}$ & $\begin{array}{l}3.658, \\
95\end{array}$ & $\begin{array}{l}0,40 \\
\%\end{array}$ & 5,5870 & 5,6315 & $0,59 \%$ & 723,89 & $\begin{array}{l}720,2 \\
0\end{array}$ & - \\
\hline ril 2021 & 3,7401 & $\begin{array}{l}3,776 \\
4\end{array}$ & $\%$ & $\begin{array}{l}3.661,7 \\
0\end{array}$ & $\begin{array}{l}3.751, \\
43\end{array}$ & $\begin{array}{l}2,53 \\
\%\end{array}$ & 5,6330 & 5,4366 & $\begin{array}{l}- \\
3,46 \%\end{array}$ & 720,45 & $\begin{array}{l}710,3 \\
0\end{array}$ & $1,37 \%$ \\
\hline $\begin{array}{r}\text { Mayo } \\
2021\end{array}$ & 3,7833 & $\begin{array}{l}3,827 \\
1\end{array}$ & $1,34 \%$ & $\begin{array}{l}3.753,3 \\
0\end{array}$ & $\begin{array}{l}3.705 \\
75\end{array}$ & $\begin{array}{l}- \\
1,22 \\
\%\end{array}$ & 5,4360 & 5,2172 & $\overline{4}, 04 \%$ & 710,75 & $\begin{array}{l}722,4 \\
3\end{array}$ & $1 \%$ \\
\hline $\begin{array}{r}\text { Junio } \\
2021\end{array}$ & 3,8344 & $\begin{array}{l}3,839 \\
7\end{array}$ & $0,33 \%$ & $\begin{array}{l}3.708,0 \\
0\end{array}$ & 0 & $\begin{array}{l}1,11 \\
\%\end{array}$ & 5,2210 & 4,9686 & - & 722,88 & $\begin{array}{l}731,7 \\
0\end{array}$ & $1,28 \%$ \\
\hline ulio 2021 & 3,8500 & $\begin{array}{l}4,058 \\
4\end{array}$ & $5,70 \%$ & $\begin{array}{l}3.750,0 \\
0\end{array}$ & $\begin{array}{l}3.874 \\
00\end{array}$ & $\begin{array}{l}3,39 \\
\%\end{array}$ & 4,9705 & 5,2 & $4,90 \%$ & 732,30 & $\begin{array}{l}756,2 \\
0\end{array}$ & $3,35 \%$ \\
\hline $\begin{array}{r}\text { Agosto } \\
2021\end{array}$ & 4,0690 & $\begin{array}{l}4,076 \\
6\end{array}$ & $0,45 \%$ & $\begin{array}{l}3.878,0 \\
0\end{array}$ & $\begin{array}{l}3.766, \\
68\end{array}$ & $\begin{array}{l}- \\
2,77 \\
\%\end{array}$ & 5,2158 & 5,1492 & - & 756,65 & $\begin{array}{l}772,6 \\
3\end{array}$ & $2,17 \%$ \\
\hline $\begin{array}{r}\text { etiembre } \\
2021\end{array}$ & 4,0920 & $\begin{array}{l}4,111 \\
8\end{array}$ & $86 \%$ & $\begin{array}{l}3.769,1 \\
5\end{array}$ & $\begin{array}{l}3.835 \\
00\end{array}$ & $\begin{array}{l}1,81 \\
\%\end{array}$ & 5,1694 & 5,2935 & $2,80 \%$ & 773,23 & $\begin{array}{l}784,8 \\
0\end{array}$ & $1,58 \%$ \\
\hline
\end{tabular}

Fuente: Elaboración propia 
DISCUSIÓN Y CONCLUSIONES:

El tipo de cambio en el Perú se moviliza de acuerdo a la oferta y demanda respecto a los dólares; es decir, es determinado por medio del régimen denominado flotación administrada. Cuando los dólares abundan en la economía, existe una tendencia a la baja con respecto al tipo de cambio y, por el contrario, cuando existe una cantidad mínima disponible de dólares, la tendencia a que el tipo de cambio aumente, se ve reflejado. Los resultados hallados evidencian que el tipo de cambio en Perú, en lo que transcurre del 2021, tiene un comportamiento enormemente voluble y que tiende al alza. Esto, principalmente se especifica, que ha sido desde el mes de abril del presente año. Algunos de los factores que reportan las fuentes que estarían involucrados en esta situación, se deberían principalmente a lo relacionado con la incertidumbre sanitaria originada a raíz de la pandemia por causa del virus Covid19 y al mismo tiempo, a la angustia política, la cual produce una serie de especulaciones. En tanto esta situación se mantenga presente en nuestro país, va a continuar siendo una alternativa la demanda por dólares, en cuanto la apreciación de la inseguridad política se acentúe. A modo de ejemplo, se podría mencionar que, de un promedio aproximado de S/ 3.63 nuevos soles (precio de dólar una semana antes de la primera vuelta electoral presidencial), este se sobrepuso a los $\mathrm{S} / 4.00$ nuevos soles a medida que el nuevo presidente del Perú, en conjunto con su gabinete, se instalaba como nuevo gobierno. La variación en el tipo de cambio, si nos referimos a un corto plazo de tiempo, responderá en cierta parte, al nivel de especulaciones al que están exhibidos los mercados cambiarios, mientras que se define por la suma de dólares que se movilizan en la economía, si hablamos de un período de tiempo de medio a largo plazo (Instituto Peruano de Economía, 2021).

Por otro lado, los especialistas hacen énfasis en el impacto de las cifras del dólar en el caso de que su valor siga aumentando y para ello mencionan que el pronóstico indica que dichas cifras no serán de gran alarma. De hecho, el BCRP, en momentos específicos, justamente participa con el fin de impedir que las variaciones vayan a ser muy rápidas o repentinas. Esto se ve reflejado en los recientes meses del año 2021, ya que, al haber intervenido, ha posibilitado el hecho de impedir un mayor aumento del tipo de cambio (Instituto Peruano de Economía, 2021). 
En el caso de Colombia, el dólar ha subido 567,49 pesos en este país durante el 2021. En lo que va del año, la moneda colombiana (peso colombiano) puede considerarse como una de las que más se ha devaluado entre los países que se han analizado. Mojica, J.L (como se citó en Diario La República, 2021) manifiesta que, últimamente, el movimiento de devaluación de la moneda colombiana se justifica a la mayor necesidad por dólares por parte de los inversionistas de todo el mundo. Uno de los factores frente a este hecho, de igual manera, es debido a la problemática e incertidumbre sanitaria y, que, a pesar de los grandes avances con respecto a la vacunación contra la Covid-19, los riesgos que se relacionan a las diferentes variantes del virus probablemente van a seguir ocasionando gran volatilidad, lo que hace que no se descarte que el tipo de cambio en Colombia, pueda seguir marcando registros elevados. Sin embargo, en cuanto las fuentes de duda se vayan aclarando, se estima que haya una corrección para los próximos meses.

Si nos referimos a Brasil, desde la llegada del Covid-19, la moneda brasileña (el real brasileño) ha sido una de las que más se ha devaluado con respecto a los países estudiados. Expertos aseguran que estas devaluaciones, bastante marcadas en las monedas en base al dólar, se deben en parte, "a la salida de flujos de capital de los mercados emergentes", a lo que el FMI (como se citó en Diario La República, 2021) valora que fue de USD 100000 millones para el período de febrero a marzo. Los datos reportados por Bloomberg (como se citó en Diario La República, 2021) manifiestan que la moneda brasileña es la divisa que reporta el peor desempeño, esto tras retroceder $1,777 \%$ durante el mes de junio de 2021.

En Chile, se ha observado una tendencia al alza con respecto al dólar. En agosto del 2021, por ejemplo, se posicionó como el segundo país en Latinoamérica con las divisas de peor desempeño, ya que el peso chileno cedió $1,452 \%$ con respecto a la moneda estadounidense, al cerrar con $\$ 749,05$. Los factores estudiados hacen ver que el precio internacional del cobre tiene influencia en este comportamiento, esto debido a que en cuanto su valor disminuye con respecto al mercado internacional, ingresa menor cantidad de divisas por medio de las exportaciones, por tanto, al hacerlas más limitadas, el valor del dólar se incrementa. Por otro lado, como menciona Campos Nélyda (2021), "anuncios de la autoridad monetaria, como la última reducción de la TPM a 3.75\%, también la afectan", esto hace que Chile sea visto como poco llamativo para los inversionistas de los demás países, algo que se 
intensifica con lo anunciado por el Sistema de Reserva Federal (FED), sobre un incremento potencial de su tasa en caso su economía siga demostrando signos de crecimiento. De esta manera, será mayor la salida de dólares del país chileno (por un aumento en la fuga de los capitales), logrando que alcen su valor, esto, mientras la diferenciación de tasas se incrementa más a beneficio de las economías extranjeras. Especialistas en el campo mencionan que se espera que esta tendencia al alza se mantenga a medida que se siga fortaleciendo el dólar a nivel internacional.

Al realizar una comparación entre el comportamiento del tipo de cambio en Perú con respecto a los demás países, podemos decir que, el devalúo del sol peruano no ha representado una cuestión alarmante a diferencia de las monedas de los países en contraste: Brasil, Colombia y Chile, lo que probablemente se explicaría por la influencia de dos tipos de factores: externos y directos. En el caso de los factores externos, nos referimos a que últimamente se ha visto una reducción de las especulaciones políticas, y con respecto a los factores directos, debido a la participación del BCRP, como anteriormente se había mencionado, con sus disposiciones estabilizadoras. Esto sumado al hecho de que los expertos en la materia hayan resaltado que en caso se dé una subida del precio del dólar, los montos se incrementarían modesta y lentamente. Por otra parte, la confianza de los ciudadanos peruanos con respecto a su moneda local, se ve reflejada, algo que ha ayudado a que signifique una divisa más constante, de esta manera, en este último período de tiempo ha sido vista una proporción menor de dólares en las cuentas de Perú.

A modo de conclusión, podemos decir que, independientemente de qué país presente porcentajes más o menos alarmantes con respeto al tipo de cambio, se ha visto que el valor del dólar viene fluctuando con mayor intensidad en lo que transcurre del 2021 en todos los países analizados, esto en consecuencia del endeudamiento a raíz de los problemas sanitarios que involucra la pandemia por el Covid-19, así como por las incertidumbres políticas y problemas internos por los que cada país atraviesa. Esto hace que las importaciones se reduzcan debido a que el panorama no está del todo claro para generar una mayor inversión de la que se está realizando. La volatilidad del dólar tiene implicancia en los países sudamericanos descritos, ya que mientras que el dólar ha subido, los precios reflejados en soles, pesos y reales, han incrementado su valor. 
REFERENCIAS BIBLIOGRÁFICAS:

Ardila, C. D. y Rincón-Castro, H. (2019). ¿Cómo y qué tanto impacta la deuda pública a las tasas de interés de mercado? Technical report, Banco de la República, Bogotá.

Arriaga, R., M. Castro y A. Rodríguez (2020), "Impactos monetarios sobre la rentabilidad del mercado accionario en México: Un análisis de cambio de régimen Markoviano." Ensayos. Revista de economía, 39(2), pp.187-21

Baillu, J., Garcés, D., Kruger, M.,y Messmacher, M. (2003). Explicación y Predicción de la Inflación en Mercados Emergentes: El Caso de México. Documento de Investigación, Bank of Canadá y Banco de México.

Balacco, H. (1986), "Algunas consideraciones sobre la definición de causalidad de Granger en el análisis econométrico". Económica, 32(2), pp. 207-255.

Baldwin, R. y Weder di Mauro, B.(eds.). (2020). Mitigating the COVID Economic Crisis: Act fast and do whatever it takes. Eds. Center of Economic Policy Research CEPR Press.

Banco Central de Reserva del Perú. (s.f.). Reporte de Inflación Setiembre 2021. https://estadisticas.bcrp.gob.pe/estadisticas/series/mensuales/resultados/PN012 $\underline{05 \mathrm{PM} / \mathrm{html} / 2021-2 / 2021-9 /}$

Barría, C. (20 de abril de 2020). Coronavirus: cómo afectará a los países más endeudados de América Latina y dónde pueden conseguir dinero para financiar el combate a la pandemia. BBC News. https://www.bbc.com/mundo/noticias-52306376

Bermúdez, E. Tipo de cambio e inflación en el Perú. [Tesis de título, Universidad Nacional de Trujillo]. Repositorio de la Universidad Nacional de Trujillo. https://dspace.unitru.edu.pe/handle/UNITRU/12770?show=full

Bollerslev, T. (1986). Generalized autoregressive conditional heteroskedasticity. Journal of Econometrics, (36),394- 419.

Bravo, H., y Garci, C. (2002). Medición de la política monetaria y el traspaso (passthrough) en Chile. Economía Chilena, 5(3). 
Brooks, C. (2019). Introductory Econometrics for Finance. Cambridge University Press.

Chung, V., (2021). Modelación de la Volatilidad del Tipo de Cambio del Dólar en el Perú: Aplicación de los Modelos GARCH y EGARCH. Revista de Análisis Económico y Financiero, 4(4), 7-12. Doi: 10.15446/ing.investig.xxxx

Clinton, K., y Perrault, J.F. (2001). Metas de inflación y tipos de cambio flexibles en economías emergentes. Estudios Económicos (7).

Domowitz, I. y Hakkio, C. (1985). Conditional variance and the risk premium in the foreign exchange market. Journal of International Economics, 19(1-2):47-66.

Dritsaki, C. (2017). An empirical evaluation in garch volatility modeling: Evidence from the stock- holm stock exchange. Journal of Mathematical Finance, (7), 366-390.

Engle, R. (1982). Autoregressive conditional heteroscedasticity with estimates of the variance of uk in ation. Econométrica, (50), 987-1007.

Flamini, A. (2007). Inflation targeting and exchangerate pass-through. Journal of International Money and Finance, 26(7), 1113-1150.

Francis, B., Hasan, I., y Hunter, D. (2002). Emerging Market Liberalization and the Impact on Uncovered Interest Rate Parity. Journal of International Money and Finance, 21, 931-956.

Gianelli Gómez, D. (2011). El traspaso de tipo de cambio a precios en Uruguay. Banco Central de Uruguay.

Groen, J. J. (2000). The monetary exchange rate model as a long-run phenomenon. Journal of International Economics, 52(2), 299-319. https:// www.doi.org/10.1016/s0022-1996(00)00061-1

Gujarati, N. and Porter, D. (2010). Econometria Basica. McGraw-Hill/Irwin, New York, 6 edition

Hurtado, I.P., y Mariño, J. (s.f). Colombia: El traspaso del tipo de cambio a los precios entre dos sistemas monetarios. 
Lozano, I. (19 de septiembre de 2021). Tipo de cambio, Perú: ¿A cuánto se cotiza el dólar? Hoy, 19 de setiembre del 2021. El Comercio. https://elcomercio.pe/economia/mercados/precio-del-dolar-en-peru-revisaaqui-cual-es-el-tipo-de-cambio-hoy-domingo-19-de-septiembre-de-2021-tipode-cambio-ocona-compra-venta-sbs-interbancario-cotizaciones-casa-decambio-nndc-noticia/

Mendonça, H. (s.f.). El real brasileño pierde la carrera contra el coronavirus. El País. https://elpais.com/economia/2020-03-19/el-real-brasileno-pierde-la-carreracontra-el-coronavirus.html

Morón, E., y Lama, R. (2006). El traspaso del tipo de cambio a precios en la economía peruana: ¿talón de Aquiles del esquema de metas de inflación? Consorcio de investigación económica y social.

Ozcelebi, O. (2018). Impacts of Exchange Rate Volatility on Macroeconomic and Financial Variables: Empirical Evidence from PVAR Modeling. In Bobek, V., editor, Trade and Global Market, Chapters. IntechOpen.

Veiga de Cabo, J., De La Fuente, E., y Zimmermann, M. (2008). Modelos de estudios en investigación aplicada: conceptos y criterios para el diseño. Medicina $y$ Seguridad del Trabajo, 54(210), 81-88.

Winkelried, D. (2012). Traspaso del tipo de cambio hacia la inflación. Estudios Económicos (23), 9-24. 